\title{
Multi-Directional Heuristic Search
}

\author{
Dor Atzmon ${ }^{1}$, Jiaoyang Li ${ }^{2}$, Ariel Felner ${ }^{1}$, Eliran Nachmani ${ }^{1}$, \\ Shahaf Shperberg $^{1}$, Nathan Sturtevant ${ }^{3}$ and Sven Koenig ${ }^{2}$ \\ ${ }^{1}$ Ben-Gurion University \\ ${ }^{2}$ University of Southern California \\ ${ }^{3}$ University of Alberta \\ dorat@post.bgu.ac.il,jiaoyanl@usc.edu, felner@bgu.ac.il, nachamni@post.bgu.ac.il, \\ shperbsh@post.bgu.ac.il, nathanst@ualberta.ca, skoenig@usc.edu
}

\begin{abstract}
In the Multi-Agent Meeting problem (MAM), the task is to find a meeting location for multiple agents, as well as a path for each agent to that location. In this paper, we introduce $\mathrm{MM}^{*}$, a MultiDirectional Heuristic Search algorithm that finds the optimal meeting location under different cost functions. $\mathrm{MM}^{*}$ generalizes the Meet in the Middle (MM) bidirectional search algorithm to the case of finding an optimal meeting location for multiple agents. Several admissible heuristics are proposed, and experiments demonstrate the benefits of $\mathrm{MM}^{*}$.
\end{abstract}

\section{Introduction}

MM [Holte et al., 2016] is a bidirectional heuristic search algorithm with a unique priority function; nodes are ordered in OPEN according to $\max (2 g, g+h)$. MM is guaranteed to meet in the middle but the practical meaning of this property is that the two search frontiers never venture further than $C^{*} / 2$ from their start locations (where $C^{*}$ is the cost of the shortest path). Nevertheless, it is important to note that a meeting location is not returned by MM; a shortest path from a start location to a goal location is returned. In fact, a meeting location is not even defined in MM.

In this paper, we deal with the "real" problem of meeting in the middle, where a meeting location is returned. Furthermore, while MM is designed for a problem that is defined on start and goal locations, our problem is defined on $k \geq 2$ start locations where we search for an optimal meeting location with respect to the shortest path from each of these $k$ start locations to that meeting location. We call this problem the Multi-Agent Meeting problem (MAM). We focus on minimizing the following two common cost functions defined on possible solutions. (1) Sum-Of-Costs (SOC): the sum of the costs of the paths to the meeting location. (2) Makespan (MKSP): the cost of the longest path to the meeting location. SOC may corresponds to the energy (fuel) consumed until the agents meet, and MKSP corresponds to the time elapsed until the agents meet. MAM has many real-life applications, such as choosing a gathering point for multiple traveling agents (humans, cars, or robots), or suggesting a location that needs to be close to important surrounding locations.
To find the optimal meeting location, we introduce the Multi-Directional Meet in the Middle (MM*) algorithm. $\mathrm{MM}^{*}$ is a best-first search algorithm that progresses in $k$ directions until a meeting location is found. We provide a unique priority function for each of the SOC and MKSP cost functions and prove the optimality of $\mathrm{MM}^{*}$ for these cases. $\mathrm{MM}^{*}$ is strongly related to $\mathrm{MM}$ - its priority function for MKSP is a generalization of that of MM, although their halting conditions are different (so as to return the actual meeting location). $\mathrm{MM}^{*}$ relies on an admissible heuristic function and we propose a number of such heuristic functions for SOC and MKSP. We then provide experimental results that demonstrate the benefits of $\mathrm{MM}^{*}$ with these heuristic functions.

MAM is also known as Optimal Meeting Point [Yan et al., 2015], Smallest Enclosing Discs [Welzl, 1991], and 1-Center problem [Megiddo, 1983] in other areas of computer science. In the field of computational geometry, MAM is known as the Weber problem [Cooper, 1968]. Many efficient algorithms exist for MAM in continuous Euclidean spaces [Ostresh Jr, 1977; Chen, 1984; Radó, 1988; Rosing, 1992] that calculate a geometric point that satisfies the relevant constraints. The problem has also been investigated on general graphs by applying variants of Dijkstra's algorithm [Dijkstra, 1959] in parallel, one for each agent. Once a path from all start locations to all locations in the graph is known, the best meeting location can be chosen by iterating over all relevant meeting locations, exhaustively. Many improvements have been suggested, such as pruning areas of the state space [Lanthier et al., 2005; Xu and Jacobsen, 2010; Yan et al., 2015; Li et al., 2019; Geisberger et al., 2008]. We continue this direction and, to the best of our knowledge, are the first ones to add admissible heuristics and study MAM in the context of heuristic search.

\section{Problem Definition}

The Multi-Agent Meeting problem (MAM) receives as input a weighted, undirected graph $G=(V, E)$ and a set of $k$ start locations $S=\left\{s_{1}, \ldots, s_{k}\right\} \subseteq V$ for $k$ agents $A=\left\{a_{1}, \ldots, a_{k}\right\}$. The cost of edge $\left(v, v^{\prime}\right) \in E$ is denoted by $c\left(v, v^{\prime}\right)>0$. A solution is a target location $t \in V$, indicating a meeting location for the agents, plus a set of shortest paths from each $s_{i}$ to $t$. Let $d(v, u)$ be the cost of a shortest 
path from $v$ to $u$. The cost functions for SOC and MKSP for a meeting location $t$ are calculated as follows. For SOC:

$$
C_{S O C}(t)=\sum_{a_{i} \in A} d\left(s_{i}, t\right) .
$$

This corresponds to the sum of the costs of the paths to the meeting location. For MKSP:

$$
C_{M K S P}(t)=\max _{a_{i} \in A} d\left(s_{i}, t\right) .
$$

This is the cost of the longest path to the meeting location. An optimal solution has the lowest cost among all possible solutions. Its cost is denoted by $C^{*}$ and its meeting location by $t^{*}$. In this paper, we focus on optimal solutions.

Figure 1(a) illustrates a MAM problem instance with three agents $a_{1}, a_{2}$, and $a_{3}$ with start locations $s_{1}, s_{2}$, and $s_{3}$, respectively. Edges are labeled with their costs. Consider location $t$ as a meeting location. Since $d\left(s_{1}, t\right)=5, d\left(s_{2}, t\right)=5$, and $d\left(s_{3}, t\right)=5, C_{S O C}(t)=15$ while $C_{M K S P}(t)=5$. Now, consider $v$. Since $d\left(s_{1}, v\right)=8, d\left(s_{2}, v\right)=2$, and $d\left(s_{3}, v\right)=2, C_{S O C}(v)=12$ and $C_{M K S P}(v)=8 . v$ is optimal for minimizing SOC and $t$ for MKSP. Next, we present the $\mathrm{MM}^{*}$ algorithm that solves MAM for both cost functions.

\section{Multi-Directional MM (MM*)}

$\mathrm{MM}^{*}$ is a multi-directional best-first search algorithm that guarantees to return an optimal MAM solution for both SOC and MKSP. As will be shown below, the only difference in $\mathrm{MM}^{*}$ between SOC and MKSP is the priority function used.

A node in $\mathrm{MM}^{*}$ is a pair $\left(a_{i}, v\right)$ representing an agent and its location. $\mathrm{MM}^{*}$ organizes nodes in a single open-list (denoted OPEN) and a single closed-list (denoted CLOSED). ${ }^{1}$ OPEN is initialized with $k$ root nodes: $\left(a_{i}, s_{i}\right)$ representing each of the $k$ agents and its start location. Each node is associated with a $g$-value. Naturally, $g\left(a_{i}, s_{i}\right)=0$. Let $N(v)$ represent the successors of $v$. Expanding a node $\left(a_{i}, v\right)$ has two parts: (1) Generating a node $\left(a_{i}, v^{\prime}\right)$ for each $v^{\prime} \in N(v)$, setting $g\left(a_{i}, v^{\prime}\right)=g\left(a_{i}, v\right)+c\left(v, v^{\prime}\right)$, and inserting it into OPEN. (2) Moving $\left(a_{i}, v\right)$ to Closed.

Recall that, in heuristic search, given a node $n$ in the search tree, $f^{*}(n)$ is defined to be the cost of the optimal solution that passes through $n$, and $f(n)$ is defined to be a lower bound on $f^{*}(n)$. This terminology is migrated to MM*. Let $f^{*}\left(a_{i}, v\right)$ be the cost of the optimal MAM solution (for either SOC or MKSP) such that $a_{i}$ passes through $v$ on its way to the meeting location. $f\left(a_{i}, v\right)$ is a lower bound on $f^{*}\left(a_{i}, v\right)$, i.e., $f\left(a_{i}, v\right) \leq f^{*}\left(a_{i}, v\right)$. In Section 4 , we define $f\left(a_{i}, v\right)$ for either SOC or MKSP by exploiting admissible heuristic functions that estimate the remaining cost of all agents (including that of $a_{i}$ ) that can be added to $g\left(a_{i}, v\right)$ (the cost of the path from $s_{i}$ to $v$ along the search tree). Each of these $f$-values can be plugged into $\mathrm{MM}^{*}$.

\footnotetext{
${ }^{1}$ Bidirectional searches usually maintain two open-lists, one for each search direction, but the priority function can choose a node from either one of them. This is logically equivalent to a single openlist which contains nodes from both directions. $\mathrm{MM}^{*}$ uses a single open-list, which is equivalent to $k$ open-lists, one for each agent, that use the same priority function.
}



There is no notion of a goal node in $\mathrm{MM}^{*}$ but instead we have a goal condition on each location $v$. We say that $v$ is a possible goal iff it has been generated from all directions, i.e., $\forall a_{i} \in A:\left(a_{i}, v\right) \in$ OPEN $\cup$ CLOSED. To manage this in practice, for each location $v$, we keep a $k$ bit-vector, where bit $i$ is set when node $\left(a_{i}, v\right)$ is generated. If $v$ is a possible goal, its cost $C(v)$ depends on the cost function and is:

$$
\begin{aligned}
& C_{S O C}(v)=\sum_{a_{i} \in A} g\left(a_{i}, v\right) \text { and } \\
& C_{M K S P}(v)=\max _{a_{i} \in A} g\left(a_{i}, v\right) .^{2}
\end{aligned}
$$

Let $U$ be the cost of the incumbent solution, i.e., $U$ is the minimum $C(v)$ among all possible goals (initially $U=\infty$ ). $U$ is an upper bound on $C^{*}$. The halting condition for MM* is to halt if $f \min \geq U$, where $\operatorname{fmin}$ is the minimum $f$-value in OPEN. This guarantees that $U$ cannot be further improved.

Algorithm 1 gives the pseudo-code of MM*. First, MM* initializes OPEN and CLOSED, and sets $U=\infty$ (line 2). Then, the initial nodes $\left(a_{i}, s_{i}\right)$ are inserted into OPEN (lines 3-4). $\mathrm{MM}^{*}$ performs a best-first search as follows. While OPEN is not empty (line 5), it extracts $\left(a_{i}, v\right)$, the best node (with the lowest $f$-value) from OPEN (line 6). Then, it checks the halting condition on location $v$, i.e., whether $f \min =$ $f\left(a_{i}, v\right) \geq U$ (lines 7-8). Otherwise, it performs the expansion cycle on $\left(a_{i}, v\right)$ (lines 9-18). MM* performs duplicate detection and pruning on CLOSED (lines 10-13) and OPEN (lines 14-16). As a result, $\mathrm{MM}^{*}$ always keeps the lowest seen $g$-value for each generated node $\left(a_{i}, v^{\prime}\right)$. In general, $\mathrm{MM}^{*}$ allows nodes in CLOSED to be re-opened (line 13). But, this will never happen for consistent heuristics (such as all heuristics that we suggest and experiment with below). If $\left(a_{i}, v^{\prime}\right)$ is not a duplicate node, then $\left(a_{i}, v^{\prime}\right)$ is inserted into OPEN (line

\footnotetext{
${ }^{2}$ In Equations 1 and 2, we used $d\left(s_{i}, v\right)$. Here, we use $g\left(a_{i}, v\right)$ because the path is the path from $s_{i}$ to $v$ along the search tree.
} 




(a)



(b)

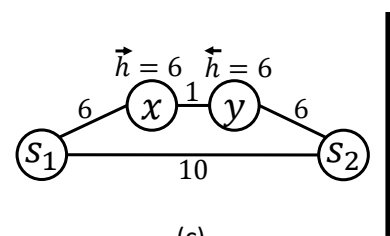

(c)

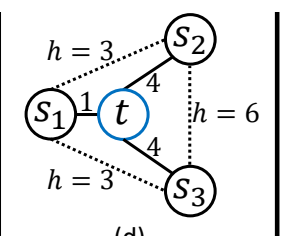

(d)

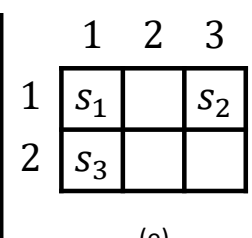

(e)

Figure 1: (a,b) $f_{M K S P}$ examples. (c) MM and $\mathrm{MM}^{*}$ difference example. (d,e) Heuristics examples.

17). Then, $U$ is updated in case $v^{\prime}$ is a possible goal with a smaller cost (line 18). After its expansion, $\left(a_{i}, v\right)$ is inserted into CLOSED (line 19). When $U$ is returned (lines 8 and 20), it also includes the meeting location $t$ as well as the paths to it, that can be constructed by parent-pointers (not included in the pseudo-code).

\section{MM* Priority Functions}

We next define the priority function $f$ for $\mathrm{MM}^{*}$ for both SOC and MKSP. In $\mathrm{A}^{*}$, given a node $n$, a perfect heuristic $h^{*}(n)$ equals $d(n, g o a l)$ and a perfect priority function $f^{*}(n)$ equals $g(n)+h^{*}(n)$. If $h(n)$ is a lower bound on $h^{*}(n)=d(n$, goal $)$, then $f(n)=g(n)+h(n)$ is a lower bound on $f^{*}(n)$. Next, we generalize this to $\mathrm{MM}^{*}$ and define all these functions for both SOC and MKSP.

\subsection{SOC}

Consider node $\left(a_{i}, v\right)$ in OPEN. $f_{S O C}^{*}\left(a_{i}, v\right)$ is the cost of the optimal solution such that: (1) $a_{i}$ passes through $v$ (via the path of cost $g\left(a_{i}, v\right)$ along the search tree). (2) $a_{i}$ continues from $v$ to meet the other agents at some location $t$. (3) Each of the other agents $a_{j}$ travels from $s_{j}$ to $t$. Now, $f_{S O C}^{*}\left(a_{i}, v\right)=$ $g\left(a_{i}, v\right)+h_{S O C}^{*}\left(a_{i}, v\right)$, where $h_{S O C}^{*}\left(a_{i}, v\right)$ is the sum of the cost of $a_{i}$ to get from $v$ to $t$ along a shortest path (item 2), plus the cost of the other agents to get from their start locations to $t$ along shortest paths (item 3 ):

$$
h_{S O C}^{*}\left(a_{i}, v\right)=\min _{t \in V}\left\{d(v, t)+\sum_{a_{j} \in A \backslash\left\{a_{i}\right\}} d\left(s_{j}, t\right)\right\} .
$$

We denote the best meeting location $t$ w.r.t. to node $\left(a_{i}, v\right)$ by $t^{*}\left(a_{i}, v\right) . h_{S O C}\left(a_{i}, v\right)$ is an admissible estimate (lower bound) of $h_{S O C}^{*}\left(a_{i}, v\right)$, i.e., $h_{S O C}\left(a_{i}, v\right) \leq h_{S O C}^{*}\left(a_{i}, v\right)$. We propose a number of admissible $h$-functions for SOC in Section 7. For SOC, naturally,

$$
f_{S O C}\left(a_{i}, v\right)=g\left(a_{i}, v\right)+h_{S O C}\left(a_{i}, v\right) .
$$

\subsection{MKSP}

The MKSP case is more complicated. Since, in MKSP, we take the maximum among agents (not the sum), we do not know which agent has the path with the highest cost. We begin by defining $f_{M K S P}^{*}\left(a_{i}, v\right)$, which is the optimal solution given that $a_{i}$ passes through $v$, via a path of cost $g\left(a_{i}, v\right)$ :

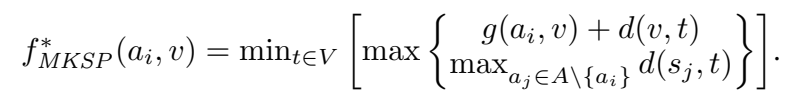

For a given possible meeting location $t$, we want the path of one of the agents with the highest cost. If this is our current agent $a_{i}$, then this is given by $g\left(a_{i}, v\right)+d(v, t)$ (top line of the max term). If it is some other agent $a_{j}$, then it is given by $d\left(s_{j}, t\right)$ (bottom line).

Next, we need to define $f_{M K S P}$ as a lower bound on $f_{M K S P}^{*}$. Here, we do not define $h_{M K S P}^{*}$ and $h_{M K S P}$ but define $f_{M K S P}\left(a_{i}, v\right)$ in terms of $h_{S O C}\left(a_{i}, v\right)$ as follows:

$$
f_{M K S P}\left(a_{i}, v\right)=\max \left\{g\left(a_{i}, v\right), \frac{g\left(a_{i}, v\right)+h_{S O C}\left(a_{i}, v\right)}{k}\right\},
$$

where $k$ is the number of agents. $g\left(a_{i}, v\right)$ is a lower bound on $f_{M K S P}^{*}\left(a_{i}, v\right)$ because $a_{i}$ has already traveled along a path of cost $g\left(a_{i}, v\right)$. Thus, $f_{M K S P}^{*}\left(a_{i}, v\right) \geq g\left(a_{i}, v\right)$. Now, observe that $\frac{f_{S O C}^{*}\left(a_{i}, v\right)}{k} \leq f_{M K S P}^{*}\left(a_{i}, v\right)$ because one of the agents must travel at least $\frac{f_{S O C}^{*}\left(a_{i}, v\right)}{k}$. Since $f_{S O C}\left(a_{i}, v\right)$ is a lower bound on $f_{S O C}^{*}\left(a_{i}, v\right)$, dividing it by $k$ will yield a lower bound on $f_{M K S P}^{*}\left(a_{i}, v\right)$.

\section{Costs of Subsets}

$f_{M K S P}^{*}$ for $k$ agents is determined by the path of one of the agents with the highest cost. Therefore, $f_{M K S P}^{*}$ and $f_{M K S P}$ for any subset of these $k$ agents are also lower bounds on $f_{M K S P}^{*}$ for all $k$ agents. Thus, for any subset of $k^{\prime}<k$ agents, we can compute $f_{M K S P}$ and use it as a lower bound on $f_{M K S P}^{*}$ for the entire set of $k$ agents. Therefore, while the right-hand side of the max function in $f_{M K S P}\left(a_{i}, v\right)$ (Equation 6) contains all $k$ agents, it can also contain any subset of agents. This can be done by calculating $h_{S O C}\left(a_{i}, v\right)$ for the selected subset of $k^{\prime}<k$ agents and dividing it by $k^{\prime}$ instead of $k$. Figure $1(\mathrm{a}, \mathrm{b})$ shows examples of MAM problem instances for MKSP. In both cases, the optimal MKSP is 5 at location $t$. Assume a perfect heuristic for SOC. For Figure 1(a), the optimal SOC is 12 at location $v$. Thus, for each agent $a_{i}, f_{S O C}\left(a_{i}, s_{i}\right)=12$, and, by computing MKSP for all agents, we get $f_{M K S P}\left(a_{i}, s_{i}\right)=12 / 3=4$. Now, consider the subset of agents $\left\{a_{1}, a_{2}\right\}$. Their SOC is 10 , and hence $f_{M K S P}\left(a_{i}, s_{i}\right)=10 / 2=5$. For Figure 1(b), the optimal SOC is 15 at location $t$, and thus $f_{S O C}\left(a_{i}, s_{i}\right)=15$. By computing MKSP for all agents, we get $f_{M K S P}\left(a_{i}, s_{i}\right)=15 / 3=$ 5 but the SOC of the subset of agents $\left\{a_{1}, a_{2}\right\}$ is 8 , and hence $f_{M K S P}\left(a_{i}, s_{i}\right)=8 / 2=4$. This shows that there is no best subset for all cases. In our experiments, we used all combinations of pairs of agents, in addition to the set of all agents. It is future work to investigate additional subset selection policies.

\section{MM \& MM* - Similarities and Differences}

It is very interesting that $f_{M K S P}$ is a generalization of the priority function of the MM algorithm: $\operatorname{pr}(n)=\max (2 g(n), g(n)+h(n))$ [Holte et al., 2016]. 
If we divide this expression by two, we get $\operatorname{pr}(n)=$ $\max \left(g(n), \frac{g(n)+h(n)}{2}\right)$. This is a special case of the proposed $f_{M K S P}$ for $k=2(h(n)$ is equivalent to an estimate of the cost from the start location of the backward agent, the goal in $\mathrm{MM}$, to the current location of the forward agent). MM prioritizes nodes based on MKSP to keep MM restrained [Shaham et al., 2017], i.e., to never expand nodes with $g(n)>\frac{C^{*}}{2}$.

In Figure 1(c), we illustrate the difference between MM and $\mathrm{MM}^{*}$ (for MKSP with 2 agents) with regard to halting. Both algorithms start by inserting $s_{1}$ and $s_{2}$ into OPEN. Next, both algorithms expand $s_{1}$ and generate $x$ and $s_{2}$. MM sets $\operatorname{pr}(x)=12$ and $\operatorname{pr}\left(s_{2}\right)=10$ (from the forward side; $h_{F}(x)=6$ and $h_{F}\left(s_{2}\right)=0$ ), and $U=10$ because a path of cost 10 has been found. At this point, MM halts, as $U \leq f \min =10$, so a path of smaller cost cannot be found. By contrast, $\mathrm{MM}^{*}$ sets $f_{M K S P}\left(a_{1}, x\right)=g\left(a_{1}, x\right)=6$ and $f_{M K S P}\left(a_{1}, s_{2}\right)=g\left(a_{1}, s_{2}\right)=10$, and $U=10$ because a meeting location with cost 10 has been found. Unlike MM, MM* continues to search because a better meeting location might be found as $f \min =f_{M K S P}\left(a_{1}, x\right)=6$. While $U$ is similar for both algorithms, the priorities of $\mathrm{MM}^{*}$ are half of the ones of MM. So, MM* continues and returns either $x$ or $y$ as meeting location. For SOC, it returns $s_{2}$ as meeting location with cost 10 , as it is on a path of minimal cost.

\section{Theoretical Analysis}

Lemma 1 (Completeness). $M M^{*}$ is guarantees to return a solution if one exists, and $U=\infty$ otherwise.

Proof outline. For each agent $a_{i}, \mathrm{MM}^{*}$ performs a best-first search from $s_{i}$. In the worst case, $\mathrm{MM}^{*}$ explores every reachable location for each agent. If a solution exists, a location reachable for all agents will be generated from all directions and $U$ will be updated. At some point, either $f \min$ will reach $U$ or the entire graph will have been explored for all agents (OPEN will be empty), and a solution will be returned. If no solution exists, there is no location that is reachable for all agents and $U$ will not be updated and thus remains $\infty$.

Lemma 2 (Optimality). Given an admissible $f$ (i.e., $f(n) \leq$ $f^{*}(n)$ for all nodes $\left.n\right), M M^{*}$ is guaranteed to return the optimal location $t^{*}$ with cost $C^{*}$.

Proof outline. Assume, by contradiction, that $\mathrm{MM}^{*}$ returned a sub-optimal location $t \neq t^{*}$ with $\operatorname{cost} C>C^{*}$. Since MM* has terminated and returned a solution, fmin $\geq C>C^{*}$. Since $\mathrm{MM}^{*}$ terminated without returning an optimal solution, there exists a node $n^{\prime}=\left(a_{i}, v_{i}\right)$ in OPEN s.t. $v_{i}$ is a location on the optimal path of $a_{i}$ to $t^{*}$, and every node before $n^{\prime}$ on the path has already been expanded. Since $n^{\prime}$ is the first node on the optimal path that was not expanded, it was generated by a node on the optimal path, and thus $g\left(n^{\prime}\right)=d\left(s_{i}, v_{i}\right)$. By definition, $f^{*}\left(n^{\prime}\right)$ is the cost of the optimal solution that passes through $n^{\prime}$. Therefore, since $g\left(n^{\prime}\right)=d\left(s_{i}, v_{i}\right)$, $f^{*}\left(n^{\prime}\right)=C^{*} . f$ is admissible. Thus, $f\left(n^{\prime}\right) \leq f^{*}\left(n^{\prime}\right)=C^{*}$. As $n^{\prime} \in$ OPEN, $f \min \leq f\left(n^{\prime}\right) \leq f^{*}\left(n^{\prime}\right)=C^{*}$, which contradicts the fact that $f \min \geq C>C^{*}$.

\section{Heuristics for MM*}

We now introduce a number of heuristics for SOC and prove their admissibility. They are plugged directly into $f_{S O C}\left(a_{i}, v\right)=g\left(a_{i}, v\right)+h_{S O C}\left(a_{i}, v\right)$ and used indirectly for $f_{M K S P}$ as shown in Equation 6. Let $t^{*}\left(a_{i}, v\right)$ be the optimal meeting location where $a_{i}$ passes through $v$. For simplicity, we use $t^{*}$ to denote $t^{*}\left(a_{i}, v\right)$ and $h\left(a_{i}, v\right)$ to denote $h_{S O C}\left(a_{i}, v\right)$. Recall that $S$ is the set of all start locations. Let $S_{i}(v)$ be the set of all start locations in $S$, except for $s_{i}$, which is replaced with $v$ (the current location of $a_{i}$ ). Formally, $S_{i}(v)=S \backslash\left\{s_{i}\right\} \cup\{v\}$. Then, $h_{S O C}^{*}\left(a_{i}, v\right)=$ $\sum_{v^{\prime} \in S_{i}(v)} d\left(v^{\prime}, \hat{t^{*}}\right)$ (from Equation 3), and we want to find a lower bound on it. $^{3}$

\section{1 $h_{1}$ : Clique Heuristic}

We assume that, for every pair of locations $\left(v_{1}, v_{2}\right)$, there exists a classic admissible heuristic $h$ (e.g., straight-line distance or Manhattan distance), such that $h\left(v_{1}, v_{2}\right) \leq$ $d\left(v_{1}, v_{2}\right)$.

Based on the triangle inequality, for every pair of locations $v_{1}, v_{2} \in S_{i}(v)$ (with $v_{1} \neq v_{2}$ ), we have that:

$$
d\left(v_{1}, v_{2}\right) \leq d\left(v_{1}, \hat{t}^{*}\right)+d\left(v_{2}, \hat{t}^{*}\right) .
$$

By summing over all such pairs, we get:

$$
\sum_{\substack{\left\{v_{1}, v_{2}\right\} \in 2^{S_{i}} \\ v_{1} \neq v_{2}}} d\left(v_{1}, v_{2}\right) \leq \sum_{\substack{\left\{v_{1}, v_{2}\right\} \in 2^{S_{i}}(v) \\ v_{1} \neq v_{2}}}\left[d\left(v_{1}, \hat{t^{*}}\right)+d\left(v_{2}, \hat{t^{*}}\right)\right] .
$$

As each $v^{\prime} \in S_{i}(v)$ is paired with $k-1$ other locations in $S_{i}(v)$, we can rewrite the right-hand side of Equation 8 as $(k-1) \cdot \sum_{v^{\prime} \in S_{i}(v)} d\left(v^{\prime}, \hat{t^{*}}\right)$. Therefore:

$$
\sum_{\substack{\left\{v_{1}, v_{2}\right\} \in 2^{S_{i}} \\ v_{1} \neq v_{2}}} \frac{d\left(v_{1}, v_{2}\right)}{k-1} \leq \sum_{v^{\prime} \in S_{i}(v)} d\left(v^{\prime}, \hat{t^{*}}\right)=h^{*}\left(a_{i}, v\right) .
$$

Now, since $h\left(v_{1}, v_{2}\right) \leq d\left(v_{1}, v_{2}\right)$, we get that:

$$
h_{1}\left(a_{i}, v\right)=\sum_{\substack{\left\{v_{1}, v_{2}\right\} \in 2^{S_{i}} \\ v_{1} \neq v_{2}}} \frac{h\left(v_{1}, v_{2}\right)}{k-1} \leq h^{*}\left(a_{i}, v\right) .
$$

This heuristic $h_{1}$ is called the Clique heuristic, as it combines the heuristic values of every (unordered) pair of locations in $S_{i}(v)$. Figure 1(d) presents an example of the clique heuristic for three agents. For node $\left(a_{1}, s_{1}\right), S_{1}\left(s_{1}\right)=\left\{s_{1}, s_{2}, s_{3}\right\}$. Therefore, $h\left(a_{1}, s_{1}\right)=$ $\frac{h\left(s_{1}, s_{2}\right)+h\left(s_{1}, s_{3}\right)+h\left(s_{2}, s_{3}\right)}{2}=\frac{3+3+6}{2}=6$.

For each start location $s_{i} \in{ }^{2} S, S_{i}\left(s_{i}\right)=S$ and hence $h_{1}$ can be calculated once for all start locations. For each location $v$ that is not a start location, all locations in $S_{i}(v)$ except for $v$ remain the same. So, $h_{1}$ can be calculated incrementally in time that is linear in the number of agents. If $h\left(v_{1}, v_{2}\right)$ is consistent, then $h_{1}$ is also consistent.

\footnotetext{
${ }^{3}$ This is a form of a front-to-end heuristic. A front-to-front heuristic needs to estimate the remaining costs when all other agents are in their current locations, but these locations thus need to be specified for a given node $\left(a_{i}, v\right)$. This is left for future work.
} 


\section{2 $h_{2}:$ Median Heuristic}

For a set of numbers $B \subset \mathbb{R}$, the median of $B$ provably minimizes the sum of the absolute deviations, i.e., $\operatorname{argmin}_{r \in \mathbb{R}} \sum_{b \in B}|b-r|=\operatorname{median}(B)$. Inspired by this property, we design the Median heuristic $\left(h_{2}\right)$ for 4-neighbor 2D grids. On such a grid, each location has two coordinates $x$ and $y$. For a set of locations, we can find the median over the $x$-coordinates (dimension 1) of all locations and the median over the $y$-coordinates (dimension 2) of all locations. Let $t m_{d}$ be the median of dimension $d$. This creates a potential meeting location $t m=\left(t m_{1}, t m_{2}\right)$, that minimizes the sum of the absolute deviations over both dimensions. Namely, if there are no obstacles on the grid, $t m$ will be the optimal meeting location for minimizing SOC. This is due to the fact that the distance between any two locations is their $L_{1}$-distance (also known as Manhattan distance on 2D grids).

Assume that the input graph $G=(V, E)$ is a 4-neighbor $2 \mathrm{D}$ grid where every location $v \in V$ is represented by its coordinates $\vec{v}=\left(v_{1}, v_{2}\right)$. The $L_{1}$-distance for any two locations $u, v \in V$ is defined as $\|\vec{u}-\vec{v}\|_{1}=\left|u_{1}-v_{1}\right|+\left|u_{2}-v_{2}\right|$. Due to the existence of obstacles, for any pair of locations $u, v \in V,\|\vec{u}-\vec{v}\|_{1} \leq d(u, v)$. So, for a given node $\left(a_{i}, v\right)$,

$$
\sum_{v^{\prime} \in S_{i}(v)}\left\|\overrightarrow{v^{\prime}}-\overrightarrow{t^{*}}\right\|_{1} \leq \sum_{v^{\prime} \in S_{i}(v)} d\left(v^{\prime}, \hat{t^{*}}\right)=h^{*}\left(a_{i}, v\right)
$$

Therefore, by modeling the problem in an empty $2 \mathrm{D} L_{1}$-space (i.e., without obstacles), we introduce a new admissible (and consistent) heuristic, called Median heuristic:

$$
\begin{aligned}
h_{2}\left(a_{i}, v\right) & =\min _{\vec{t} \in \mathbb{R}^{2}}\left\{\sum_{v^{\prime} \in S_{i}(v)}\left\|\overrightarrow{v^{\prime}}-\vec{t}\right\|_{1}\right\} \\
& =\sum_{v^{\prime} \in S_{i}(v)}\left\|\overrightarrow{v^{\prime}}-t \vec{m}\right\|_{1} \\
& =\sum_{v^{\prime} \in S_{i}(v)}\left[\left|v_{1}^{\prime}-t m_{1}\right|+\left|v_{2}^{\prime}-t m_{2}\right|\right] .
\end{aligned}
$$

It is admissible because the right-hand side of Equation 12 is no larger than the left-hand side of Equation 11.

We use the Quick-select algorithm for finding medians [Hoare, 1961], which runs in $\Theta(k)$ time, to compute $h_{2}\left(a_{i}, s_{i}\right)$ for all root nodes. Then, for every non-root node $\left(a_{i}, v\right)$ (i.e., $\left.v \neq s_{i}\right), k-1$ locations have not changed, and we only need to update the median based on the single location that has changed. This can be done in $O(1)$ time.

Figure 1(e) shows an example of a MAM problem instance with three agents $\left(s_{1}, s_{2}\right.$, and $\left.s_{3}\right)$ that are located in $(1,1),(3,1)$, and $(1,2)$. The $x$-coordinates are $\{1,3,1\}$ and the $y$-coordinates are $\{1,1,2\}$. Thus, the median location is $(1,1)$. By computing the Manhattan distances from $(1,1)$ to each start location, we get $h_{2}\left(a_{i}, s_{i}\right)=3$ for each agent $a_{i}$.

$h_{2}$ can be generalized easily for 6-neighbor $3 \mathrm{D}$ grids and similar graphs of higher dimensions.

\section{3 $h_{3}$ : FastMap Heuristic}

The Median heuristic $\left(h_{2}\right)$ only works for graphs that have coordinates. In addition, $h_{2}$ ignores obstacles, which may introduce inaccuracies to the heuristic. The FastMap heuristic $\left(h_{3}\right)$ handles this. FastMap [Cohen et al., 2018; Li et al., 2019] is a near-linear preprocessing algorithm that embeds the locations of a given edge-weighted undirected connected graph $G=(V, E)$ into a $D$-dimensional $L_{1}$-space $\mathbb{R}^{D}$. The dimension $D$ of the $L_{1}$-space is user-specified. Each location $v_{i} \in V$ is mapped to a $D$-dimensional point $\overrightarrow{p_{i}} \in \mathbb{R}^{D}$. The length of a shortest path $d\left(v_{i}, v_{j}\right)$ between any two locations $v_{i}, v_{j} \in V$ is approximated by the $L_{1}$-distance $\left\|\overrightarrow{p_{i}}-\overrightarrow{p_{j}}\right\|_{1}$ between the corresponding two points $\overrightarrow{p_{i}}, \overrightarrow{p_{j}} \in \mathbb{R}^{D}$ in this space. FastMap ensures that the $L_{1}$-distance in $\mathbb{R}^{D}$ can be used as an admissible and consistent heuristic for the shortest path computation in $G$. See [Cohen et al., 2018] for more details on FastMap. To compute $h$-values for MAM, $h_{3}$ applies the Median heuristic on the generated embedding $\mathbb{R}^{D}$. For any node $\left(a_{i}, v\right)$, its FastMap heuristic is defined as:

$$
h_{3}\left(a_{i}, v\right)=\min _{\vec{t} \in \mathbb{R}^{D}}\left\{\sum_{v^{\prime} \in S_{i}(v)}\left\|\overrightarrow{p^{\prime}}-\vec{t}\right\|_{1}\right\},
$$

where $\overrightarrow{p^{\prime}} \in \mathbb{R}^{D}$ is the point of the embedding of location $v^{\prime}$ generated by FastMap. By the same analysis as for $h_{2}$, we can show that the FastMap heuristic $\left(h_{3}\right)$ is admissible (and consistent), and we can compute it for every root node in $\Theta(k D)$ time and for any non-root node in $O(D)$ time.

There are many other approaches for embedding a graph in a continuous space, such as [Ng and Zhang, 2002; Shavitt and Tankel, 2004; Rayner et al., 2011]. However, most of them use $L_{2}$-distances, which are not applicable here because the $L_{2}$-distance version of Equation 15 is NP-hard to solve optimally [Hoare, 1961].

\section{Experimental Results}

We experimented with MM* on an Intel $\mathrm{R}$ Xeon E5-2660 v4@2.00GHz processor with 16GB of RAM. We compared all new heuristics to the Dijkstra version of $\mathrm{MM}^{*}$, i.e. where $h=0$ (denoted by $h_{0}$ ), on different grids while minimizing both SOC and MKSP. For $h_{1}$, we used the Manhattan Distance (MD) as a classic admissible heuristic between any two locations. The number of dimensions $D$ for $h_{3}$ was always set to 10, as suggested by Li et al. [2019].

\subsection{SOC}

We experimented on a $500 \times 500$ grid with $0 \%-30 \%$ obstacles. Table 1(left) shows the average over 50 instances of: the solution cost, the initial $h$-value, the number of expansions, and the CPU time for 5 randomly placed agents. $h_{2}$ had the best initial $h$-value, had the lowest number of expansions, and was the fastest. The initial $h$-values of $h_{1}(\mathrm{MD})$ and $h_{2}$ remain constant as more obstacles are added since they both ignore obstacles. By contrast, $h_{3}$ increases as more obstacles are added. So, $h_{2}$ degrades while $h_{3}$ improves, in terms of number of expansions and CPU time. $h_{3}$ incurred a preprocessing time of $\approx 30$ s, which is incurred only once per grid and thus amortized over multiple problem instances.

We also fixed the number of obstacles at $10 \%$ while varying the number of agents from 3 to 9. Table 2(left) shows the CPU time for SOC. Here, too, $h_{2}$ was the best heuristic with only $1.27 \mathrm{~s}$ for 9 agents because $h_{2}$ is suitable for grids with small numbers of obstacles. 




Figure 2: (a) Enigma grid. (b) SOC expansions. (c) SOC time. (d) MKSP expansions. (e) MKSP time.

\begin{tabular}{|c|c|c|c|c|c|c|c|c|}
\hline & \multicolumn{4}{|c|}{ SOC } & \multicolumn{4}{|c|}{ MKSP } \\
\hline \#Obs. & $0 \%$ & $10 \%$ & $20 \%$ & $30 \%$ & $0 \%$ & $10 \%$ & $20 \%$ & $30 \%$ \\
\hline \multicolumn{9}{|c|}{ Cost } \\
\hline & 995 & 1,008 & 1,033 & 1,103 & 292 & 293 & 295 & 305 \\
\hline \multicolumn{9}{|c|}{ Initial $h$-value } \\
\hline$h_{0}$ & 0 & 0 & 0 & 0 & 0 & 0 & 0 & U \\
\hline$h_{1}$ & 828 & 828 & 828 & 828 & 166 & 166 & 166 & 166 \\
\hline$h_{2}$ & 995 & 995 & 995 & 995 & 199 & 199 & 199 & 199 \\
\hline$h_{3}$ & 643 & 680 & 736 & 778 & 127 & 137 & 140 & 148 \\
\hline \multicolumn{9}{|c|}{ \#Expansions (thousands) } \\
\hline$h_{0}$ & 1,244 & 1,120 & 994 & 856 & 542 & 485 & 420 & 341 \\
\hline$h_{1}$ & 330 & 322 & 320 & 318 & 180 & 15 & 33 & 121 \\
\hline$h_{2}$ & 34 & 58 & 83 & 143 & 179 & 158 & 132 & 119 \\
\hline$h_{3}$ & 634 & 561 & 465 & 402 & 308 & 299 & 239 & 197 \\
\hline \multicolumn{9}{|c|}{ Time $(\mathrm{s})$} \\
\hline$h_{0}$ & 22.05 & 19.04 & 16.10 & 13.04 & 7.81 & 6.69 & 5.47 & 4.10 \\
\hline$h_{1}$ & 4.50 & 4.29 & 4.18 & 4.01 & 2.83 & 2.43 & 1.94 & 1.66 \\
\hline$h_{2}$ & 0.28 & 0.54 & 0.77 & 1.40 & 2.79 & 2.40 & 1.93 & 1.64 \\
\hline$h_{3}$ & 9.48 & 8.66 & 6.81 & 5.60 & 6.99 & 5.44 & 4.12 & 4.00 \\
\hline
\end{tabular}

Table 1: Results on $500 \times 500$ grids with varying obstacles.

Finally, we experimented on the $768 \times 768$ Enigma grid (presented in Figure 2(a)) from the Starcraft video game, available in the movingai repository [Sturtevant, 2012]. Figures 2(b) and 2(c) show the average number of expansions and CPU time, respectively, for 3 to 9 agents for SOC. Here, $h_{3}$ was the best heuristic in both expansions and time. Since this grid has many obstacles (about 57\%), $h_{1}$ and $h_{2}$ were less effective than $h_{3}$, which uses real distances (albeit in the embedded graph). Nevertheless, $h_{3}$ required a preprocessing time of 39s for this grid (done once). $h_{2}$ was the second best heuristic but $h_{2}$ is only suited for grids while $h_{1}$ can be used on any graph. For 9 agents, $h_{1}$ expanded slightly fewer nodes than $h_{0}$ but, since it consumes time for computing the heuristic, was a little slower than $h_{0}$.

\subsection{MKSP}

As described in Section 4, there are different policies for choosing subsets for computing MKSP. We compared two of these policies: (1) selecting all agents and (2) selecting all agents plus all pairs of agents and taking the maximum over all of those. Figure 3 shows the number of expansions and

\begin{tabular}{|c|c|r|r|r||c|c|r|r|}
\hline & \multicolumn{5}{|c||}{ SOC } & \multicolumn{4}{c|}{ MKSP } \\
\hline \#Agents & \multicolumn{1}{|c|}{5} & \multicolumn{1}{|c|}{7} & \multicolumn{1}{c|}{9} & 3 & \multicolumn{1}{c|}{5} & \multicolumn{1}{c|}{9} \\
\hline$h_{0}$ & 6.87 & 18.98 & 29.46 & 44.17 & 1.91 & 6.73 & 11.03 & 18.07 \\
$h_{1}$ & $\mathbf{0 . 1 6}$ & 4.66 & 16.15 & 36.29 & 0.47 & 2.62 & 3.57 & 6.53 \\
$h_{2}$ & $\mathbf{0 . 1 6}$ & $\mathbf{0 . 8 1}$ & $\mathbf{0 . 8 5}$ & $\mathbf{1 . 2 7}$ & $\mathbf{0 . 4 6}$ & $\mathbf{2 . 6 0}$ & $\mathbf{3 . 5 2}$ & $\mathbf{6 . 3 8}$ \\
$h_{3}$ & 1.92 & 8.50 & 18.66 & 33.20 & 1.48 & 6.57 & 9.59 & 16.46 \\
\hline
\end{tabular}

Table 2: Avg. time (s) on $500 \times 500$ grids with $10 \%$ obstacles
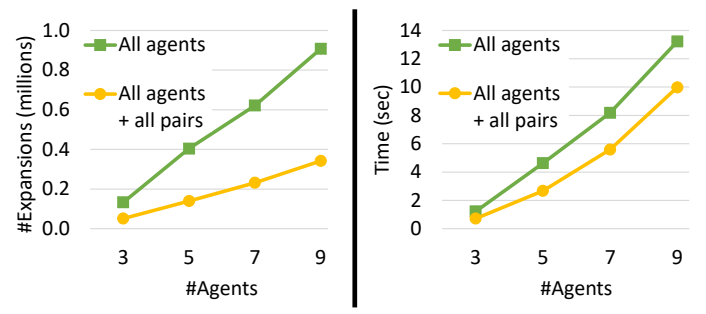

Figure 3: MKSP results for $h_{3}$ on the Enigma grid.

CPU time averaged over 50 instances for $h_{3}$ on the Enigma grid with 3 to 9 agents. As expected, adding all pairs of agents produces a better heuristic and fewer expansions. It was also better in terms of the CPU time despite the fact that its computation overhead is larger. Therefore, we used this subset selection policy for computing MKSP. It is future work to investigate different policies for choosing subsets.

We repeated the same experiments (reported for SOC) for MKSP. Table 1(right) presents the results. Here, too, $h_{2}$ was the best heuristic but here (unlike SOC) $h_{1}$ was very close to $h_{2}$. This is so probably because the clique heuristic $\left(h_{1}\right)$ for MKSP also guides the agents to the median.

The same trends were observed for different numbers of agents with $10 \%$ obstacles and are presented in Table 2(right).

In the Enigma grid for MKSP (Figures 2(d,e)), $h_{3}$ was again the best heuristic. $h_{1}$ and $h_{2}$ (curves cover each other) had fewer numbers of expansion but were slower than $h_{0}$.

\section{Conclusions}

We introduced the multi-directional search algorithm $\mathrm{MM}^{*}$ that optimally solves MAM. We proved that $\mathrm{MM}^{*}$ is complete and optimal and suggested a few admissible heuristics. Experimentally, we showed that $\mathrm{MM}^{*}$ performs better with heuristics. For grids with few obstacles, $h_{2}$ is best. For grids with many obstacles, $h_{3}$ is best but requires preprocessing. The advantage of $h_{1}$ is that it is applicable to all domains without the need for preprocessing. Future work will: (1) develop a weighted version of $\mathrm{MM}^{*}$ that can find boundedsuboptimal solutions, (2) extend MM* to a Euclidean space, and (3) further investigate subset selection for MKSP.

\section{Acknowledgements}

This research was supported by ISF grant $844 / 17$ and BSF grant 2017692 to Ariel Felner, NSF grant 1815660 to Nathan R. Sturtevant and NSF grants 1409987, 1724392, 1817189, 1837779 and 1935712 to Sven Koenig. 


\section{References}

[Chen, 1984] Reuven Chen. Location problems with costs being sums of powers of Euclidean distances. Computers \& Operations Research, 11(3):285-294, 1984.

[Cohen et al., 2018] L. Cohen, T. Uras, S. Jahangiri, A. Arunasalam, S. Koenig, and T. K. S. Kumar. The FastMap algorithm for shortest path computations. In the International Joint Conference on Artificial Intelligence (IJCAI), pages 1427-1433, 2018.

[Cooper, 1968] Leon Cooper. An extension of the generalized Weber problem. Journal of Regional Science, 8(2):181-197, 1968.

[Dijkstra, 1959] Edsger W Dijkstra. A note on two problems in connexion with graphs. Numerische Mathematik, 1(1):269-271, 1959.

[Geisberger et al., 2008] Robert Geisberger, Peter Sanders, Dominik Schultes, and Daniel Delling. Contraction hierarchies: Faster and simpler hierarchical routing in road networks. In the International Workshop on Experimental and Efficient Algorithms, pages 319-333, 2008.

[Hoare, 1961] Charles AR Hoare. Algorithm 65: Find. Communications of the ACM, 4(7):321-322, 1961.

[Holte et al., 2016] Robert C. Holte, Ariel Felner, Guni Sharon, and Nathan R. Sturtevant. Bidirectional search that is guaranteed to meet in the middle. In the $A A A I$ Conference on Artificial Intelligence (AAAI), pages 34113417, 2016.

[Lanthier et al., 2005] Mark A Lanthier, Doron Nussbaum, and Tsuo-Jung Wang. Calculating the meeting point of scattered robots on weighted terrain surfaces. In the Australasian Theory Symposium, volume 41, pages 107-118, 2005.

[Li et al., 2019] Jiaoyang Li, Ariel Felner, Sven Koenig, and T. K. S. Kumar. Using FastMap to solve graph problems in a Euclidean space. In the International Conference on Automated Planning and Scheduling (ICAPS), pages 273$278,2019$.

[Megiddo, 1983] Nimrod Megiddo. The weighted Euclidean 1-center problem. Mathematics of Operations Research, 8(4):498-504, 1983.

[Ng and Zhang, 2002] T. S. Eugene Ng and Hui Zhang. Predicting internet network distance with coordinates-based approaches. In the Annual Joint Conference of the IEEE Computer And Communications Societies (INFOCOM), pages 170-179, 2002.

[Ostresh Jr, 1977] Lawrence M Ostresh Jr. The multifacility location problem: Applications and descent theorems. Journal of Regional Science, 17(3):409-419, 1977.

[Radó, 1988] Francisc Radó. The Euclidean multifacility location problem. Operations Research, 36(3):485-492, 1988.

[Rayner et al., 2011] Chris Rayner, Michael Bowling, and Nathan R. Sturtevant. Euclidean heuristic optimization. In the AAAI Conference on Artificial Intelligence (AAAI), pages 81-86, 2011.
[Rosing, 1992] Kenneth E Rosing. An optimal method for solving the (generalized) multi-Weber problem. European Journal of Operational Research, 58(3):414-426, 1992.

[Shaham et al., 2017] Eshed Shaham, Ariel Felner, Jingwei Chen, and Nathan R. Sturtevant. The minimal set of states that must be expanded in a front-to-end bidirectional search. In the International Symposium on Combinatorial Search, SoCS, pages 82-90, 2017.

[Shavitt and Tankel, 2004] Yuval Shavitt and Tomer Tankel. Big-bang simulation for embedding network distances in Euclidean space. IEEE/ACM Transactions on Networking, 12(6):993-1006, 2004.

[Sturtevant, 2012] Nathan R. Sturtevant. Benchmarks for grid-based pathfinding. Computational Intelligence and AI in Games, 4(2):144-148, 2012.

[Welzl, 1991] Emo Welzl. Smallest enclosing disks (balls and ellipsoids). In New Results and New Trends in Computer Science, pages 359-370. Springer, 1991.

[Xu and Jacobsen, 2010] Zhengdao Xu and Hans-Arno Jacobsen. Processing proximity relations in road networks. In the ACM SIGMOD International Conference on Management of Data (SIGMOD), pages 243-254, 2010.

[Yan et al., 2015] Da Yan, Zhou Zhao, and Wilfred Ng. Efficient processing of optimal meeting point queries in Euclidean space and road networks. Knowledge and Information Systems, 42(2):319-351, 2015. 